\title{
WAVES AND OSCILLATIONS IN THE NON-MAGNETIC PHOTOSPHERE
}

\author{
Franz - Ludwig Deubner \\ Institut für Astronomie und Astrophysik \\ der Universität Würzburg \\ Am Hubland, D-8700 Würzburg
}

\section{Introduction}

The solar photosphere is the outer boundary of the cavity which defines the spectrum of the global p-modes. It is also the lowest layer that permits direct observation of the boiling mixture of motions which eventually create the complex patterns apparent in the chromosphere and corona. Studies of the dynamical behaviour of this layer are therefore of paramount importance for an overall understanding of the dynamic sun.

Although the basic hydrodynamic processes occurring in and close to the "quiet" photosphere were described more than two decades ago, the verification of its detailed dynamic behaviour by observation created a complex puzzle some important pieces of which have only lately fallen into their proper places, while others, surprisingly, are still being added.

It is impossible, in the frame of this review, to give a fair and full account of the immense volume of observational accomplishments in the field of solar atmospheric dynamics achieved since, say, the classical report by Noyes (1967), when the 5-min oscillations were not yet recognized as a phenomenon of the solar interior. The interested reader is referred to more recent reviews by Frandsen (1988), and by Deubner et al. (1984).

After the discovery of the global coherence of the solar p-modes motions and waves in the visible atmosphere were studied for three major purposes, apart from Helioseismology:

(1) The heating of the outer layers of the atmosphere; in the quiet sun acoustic and gravity waves are still considered prime candidates for carriers of non-thermal energy, at least in the low chromosphere. Yet, a conclusive quantitative account of the contributions from various competitive MHD and non-MHD processes is not available. However, this challenging topic is outside the scope of this article.

(2) Investigations of stellar convection; measurements of motions and brightness distributions of granulation, meso- and supergranulation reveal basic physical properties of convection, against which theory and models need to be checked, before they can be confidently applied to the invisible interior of any star.

(3) The main emphasis of this review will be on spectral diagnostics of oscillations and waves. Power, phase and coherence spectra serve to define the character of a wave - propagating or evanescent, standing or running - as they can be easily related to the relevant dispersion relations, and to explore the physical properties of the medium that supports oscillatory motions. The latter aspect is particularly important for our understanding of the structure of the dynamical atmosphere. 
In the following chapter we shall very briefly review recent observational work describing the interaction of granulation and waves. Chapters 3 to 5 will center around some unresolved issues arising from new results of observational photospheric oscillation studies. It appears that the k- $\omega$ spectra of phase and coherence, while improving the wave diagnostics dramatically, are also good for intriguing surprises.

\section{Low frequency waves and convection}

The art of modelling solar (and stellar) convection by simulation calculations (Nordlund, 1984; Stein et al., 1989; Steffen et al., 1989) has now reached a degree of perfection, that permits direct comparison of observable parameters (brightness contrast, r.m.s. velocities, line width variations, bisectors etc.) with corresponding values derived from the models (Dravins et al., 1981; Wöhl and Nordlund, 1985; Steffen, 1989). However, there are still severe geometrical restrictions to those models; owing to the limited memory space in computers the volume studied in 3-D simulations typically includes no more than a few granules, and consequently the discussion of larger scale structures such as mesogranulation is mostly based on similarity arguments. Due to these circumstances there is (fortunately) still much room for fundamental observational contributions.

To begin with the larger scale phenomena, there is first the simple question, whether mesogranulation indeed constitutes a distinct regime of convective motions separated from granulation and from supergranulation by well defined gaps in the spatial velocity or intensity power spectra. Some evidence in favour of such a picture was recently presented and discussed by Deubner (1989), who finds enhanced power in the velocity and brightness distribution in a range of scales between 5 " and 10"; but a firm conclusion on this issue may require an investigation of two-dimensional data. In fact, this seemingly simple question entails a rather difficult observational task: High spatial resolution (and very good seeing!) is needed as well as good wave number separation, i.e. a large field of view; since the velocity signal changes its structure and amplitude with distance from the disc center due to perspective, one must probably be satisfied with the rather low contrast brightness signal for the low wave number section of the spatial power spectra; the duration of the observation should comply with the typical time scales of the larger spatial scale phenomena (at least several hours), and with the necessity to separate them in frequency space from waves and oscillations.

A second question concerns the dynamics of solar convection near the photosphere, and of mesogranulation in particular. If mesogranulation is indeed convective in origin, as is generally assumed, the vertical flow pattern observed by November et al. (1981) should be correlated with the brightness distribution, as in the case of granules. Mesoscale brightness fluctuations have been observed as early as 1932 by Strebel and Thüring, and again by Koutchmy and Lebecq (1986), and by Oda (1984); a high degree of correlation with the flow pattern is confirmed by Deubner's (1989) analysis. The temporal phase differences observed between velocity fluctuations at different levels of the atmosphere, and between velocity and brightness fluctuations in various spectral lines as function of the spatial scale testify strongly for the convective origin of the observed quasi cellular motions at all spatial scales in the photosphere, and of mesogranules in particular.

The mesoscale horizontal velocities inferred from our power spectra are of the order of $750 \mathrm{~m} / \mathrm{s}$. They are in good agreement with the r.m.s. values derived from high resolution observations of granule proper motions (November et al., 1987; Brandt et al., 1988). This comparison justifies the previous assumption, that the observed proper motions represent in fact the horizontal bulk velocities of small scale convective elements. The observed vertical motions are 
fairly strong $(-300 \mathrm{~m} / \mathrm{s})$ and obviously sufficient to suppress, impede or accelerate the evolution of individual granules, as seen in white light movies (Brandt et al., 1989). From Deubner's study follows also, that the observed ratio of vertical to horizontal flow velocity of convective motions depends nearly linearly on the spatial scale, being on the order of 1 for granules, 2.6 for mesogranules and about 20 for supergranules. In cylindrical geometry the continuity equation does indeed suggest such a linear scaling law.

A third area of interest is the overshoot region and the interaction of convection with the stable layers on top. The early observational finding by Evans and Catalano (1972), namely the inversion of the granular brightness contrast at a hight of approximately $100 \mathrm{~km}$, has been confirmed by many observers, and is clearly present also in the simulations of Nordlund (....) and Steffen et al. (1989) as a consequence of the adiabatic expansion of overshooting elements into the stably stratified atmosphere. This is not the whole story, however, and the actual distribution of brightness and velocity relates in a very complex way to the convective pattern underneath. In a statistical study of the intensity fluctuations and Doppler shifts measured in individual high spatial resolution spectrograms, Nesis et al. (1988) find a rapid decay of the spatial correlation of the observed structures with height, suggestively described as a "loss of memory". An unknown independent velocity field is invoked to explain the observational results. Let us have a look at the kind of motions which might perturb a quasi stationary velocity and brightness distribution.

If these disturbations somehow formed a coherent pattern they should become apparent in a Fourier decomposition of the velocity or brightness data with regard to frequency and wavenumber, i.e. in k- $\omega$ diagrams of power or temporal phase lag. The SOUP experiment on the Spacelab 2 shuttle flight (Title et al., 1986) detected f-modes up to $l=3500$ (1."7). In this wavenumber regime confusion with the velocity and brightness distribution of granulation is very likely, even if at this $l$ value the fundamental mode period $(-200 \mathrm{~s})$ is considerably shorter than the granular lifetime. At lower frequencies $(v \leq 4 \mathrm{mHz})$, and for $l \geq 1400$ Deubner and Fleck (1989) have demonstrated the presence of propagating internal gravity waves in the photosphere by comparing the phase lag between the Doppler shifts measured at different heights in the atmosphere. Locally coherent evanescent oscillations are present in several other areas of the $\mathrm{k}-\omega$ diagram as we shall discuss in Chapter 3 and 4.

The relation between any of these wave fields and granular or mesogranular motions has not been studied in detail yet. Therefore, the question which of these motions and corresponding brightness fluctuations are indeed generated independently from the convective processes, and which ones occur always in the wake of granules can not be answered from the observational point of view at the present time.

\section{Evanescent waves of various kinds}

In an adiabatic stratified atmosphere, theory predicts a phase difference between velocity and temperature fluctuations of $90^{\circ}$ in the evanescent regime, and an asymptotic approach to $0^{\circ}$ at frequencies beyond the acoustic cut off. With thermal relaxation taken into account the phase in the evanescent regime tends to higher values, and should become $180^{\circ}$ in the fully isothermal case. This behaviour was first studied extensively both observationally and in fundamental theoretical investigations by Schmieder $(1976,1977,1978)$, and later by Lites and Chipman (1979), who confirmed Schmieder's observations.

Within certain limits, to be discussed in Chapter 5, those observations are in accordance with theoretical calculations for frequencies higher than $\sim 2.5 \mathrm{mHz}$. But, in Lites and Chipman's (1979) Figure 1, a surprising phase discontinuity is observed at approximately 2.2 
$\mathrm{mHz}$ which is clearly visible also in every single photospheric V-I phase spectrum displayed in Staiger's (1985) thesis. We present a collage of Staiger's observations in Figure 1.

Gravity waves can not be responsible for the discontinuity and the negative phases found between 1 and $2 \mathrm{mHz}$, because the V-I phases of gravity waves are rather similar to those of evanescent oscillations at the observed frequencies (Mihalas and Toomre, 1981). Superposition of evanescent waves with granular impulsive motions was therefore suggested as an alternative explanation (Lites and Chipman, 1979), although the discontinuity appears most conspicuously in the phase spectra of lines formed near the temperature minimum, and the absolute value of the maximum phase difference $\left(50^{\circ}-60^{\circ}\right)$ depends very little on the height of the line forming layer.

Decomposition of the phase difference spectra with respect to frequency and wavenumber (Deubner and Fleck, 1989, Figure 6) revealed that the highest positive phase values are connected to fluctuations observed at wavenumbers with a spatial scale larger than 5". The presence of convective motions at this scale (corresponding to the mesogranular pattem discovered by November et al., 1981) has indeed been demonstrated by Deubner (1989); however, the case for this particular regime of convective motions causing the strange phase discontinuity did not appear very convincing.

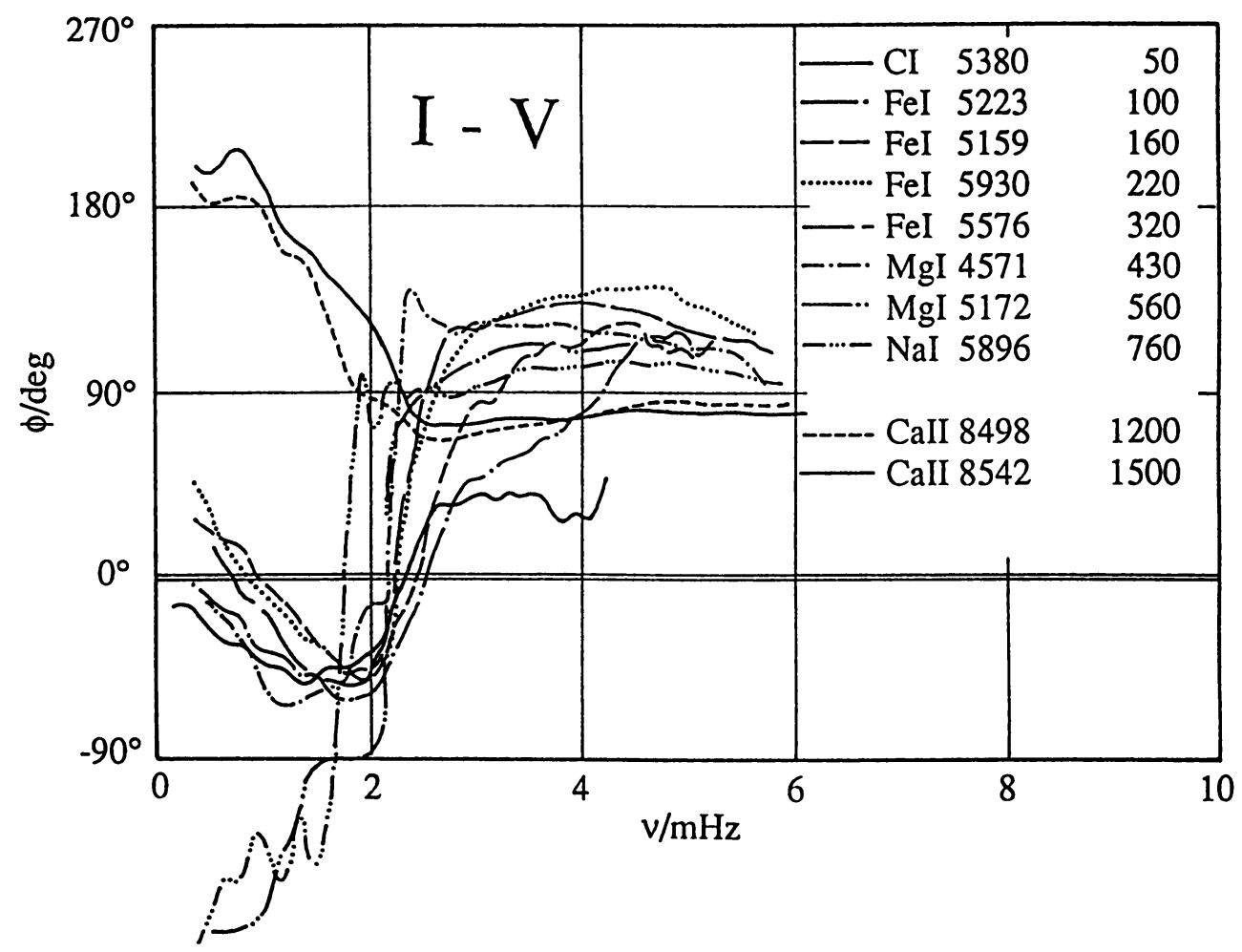

Fig. 1. Brightness - velocity phase spectra compiled from Staiger's (1985) thesis work. Positive phase indicates that brightness lcads upward velocity. Approximatc line formation heights are given in $\mathrm{km}$. 
At this stage, Marmolino and Severino (1990) presented theoretical phase difference diagrams in the $\mathbf{k}-\omega$ plane, based on an isothermal model atmosphere with adjustable thermal relaxation. These diagrams indicated clearly, that the observed discontinuity is not related to the presence of large scale convective motions, but follows directly from the dispersion relation of waves in a stratified atmosphere. In fact, there are two such discontinuities present in the k$\omega$ diagram, enclosing a trough with a triangular base (in the double logarithmic presentation of Marmolino and Severino, this conference) dubbed the "missing piece of cake" for its shape.

The position of the two discontinuities (i.e. the walls of the trough) correspond (a) to the linear dispersion relation of Lamb waves (horizontally propagating sound waves with $\omega=k V_{s}$ ) for the low frequency flank, and (b) to the dispersion relation of divergence free waves $\left(\omega^{2}=g k\right)$ for the high frequency flank. In the latter case the locus of the discontinuity is characterized by a minimum of the pressure disturbation; the other discontinuity occurs where the vertical velocity component of the wave field has a minimum. According to this theoretical diagram we should compare the observed discontinuity in the frequency spectra (Figure 1) with case (b), since only here the phase jump goes in the right direction. The inverse slope of the phase at lower frequencies $(<1.5 \mathrm{mHz})$ would then have to be associated with case (a).

Can the counterparts of the "missing piece" be seen directly in observed $\mathbf{k}-\omega$ diagrams? The aforementioned Figure 6 in Deubner and Fleck (1989) does not fully match the theoretical diagram, because the data are only one-dimensional in the spatial coordinate, $k_{x}$. In Figure 2 we present the results of a recent study in the form of $k-\omega$ diagrams, where the horizontal wavenumber $\mathbf{k}$ was deduced from two-dimensional data. Now the $\mathrm{p}$-mode ridges can be well recognized in the crosspower diagram in the lower left. The lowest one is the $\mathrm{f}$ - or divergence free mode. The Lamb waves are indicated by the slanting straight line. V-I phases are coded by two sets of grey levels which differ by $60^{\circ}$ as indicated in the right column next to the phase diagrams.

In the high wavenumber regime, the phase diagrams are obviously dominated by noise and seeing effects, pulling the phase towards zero. Below the Lamb line the phase is negative (approximately $-30^{\circ}$ ), and positive (approximately $+90^{\circ}$ ) directly above it, in qualitative agreement with the theoretical model of a nearly adiabatic gas. Averaging of all spatial scales yields a gradually increasing contribution of positive phases with increasing frequency. This explains the smooth transition from positive to negative values in the 0 to $1.5 \mathrm{mHz}$ range of the onedimensional frequency spectra derived for the photospheric lines in Figure 1. (Figures 1 and 2 have opposite sign conventions!) The f-mode on the other hand appears in the diagram as a ridge with a distinct negative $\left(\sim-90^{\circ}\right)$ phase rather than as a cliff. The counterpart of the high frequency edge of the piece of cake apparently is not formed by the dividing line between "gravity like" and "pressure like" waves, but by the low frequency envelope of the power distribution of the low order p-modes. Again, averaging of the spatial coordinate readily explains the existence of a sharp ridge at $-2.2 \mathrm{mHz}$ in the frequency spectra.

Alas! This finding does not concur in all aspects with theoretical expectation. It appears that more realistic simulations of atmospheric waves, including the global modes, are necessary to reproduce the observational results.

Our data indicate the presence of solar noise power in the range of frequencies occupied by low order global p- and g-modes. It is difficult to estimate its true amplitude because of the superposed effects of instrumental trends and seeing revealed by the coherence spectrum in Figure 2. Nevertheless, one needs to be aware of the possibility, that these helioseismologically most important modes are contaminated by a kind of noise, which can not be avoided by space experiments. 

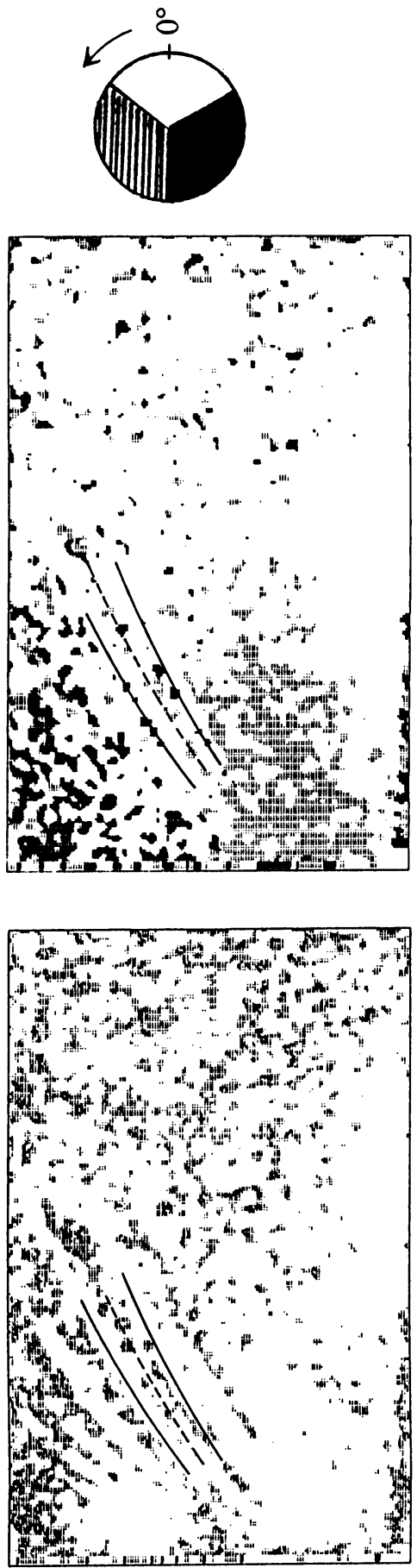
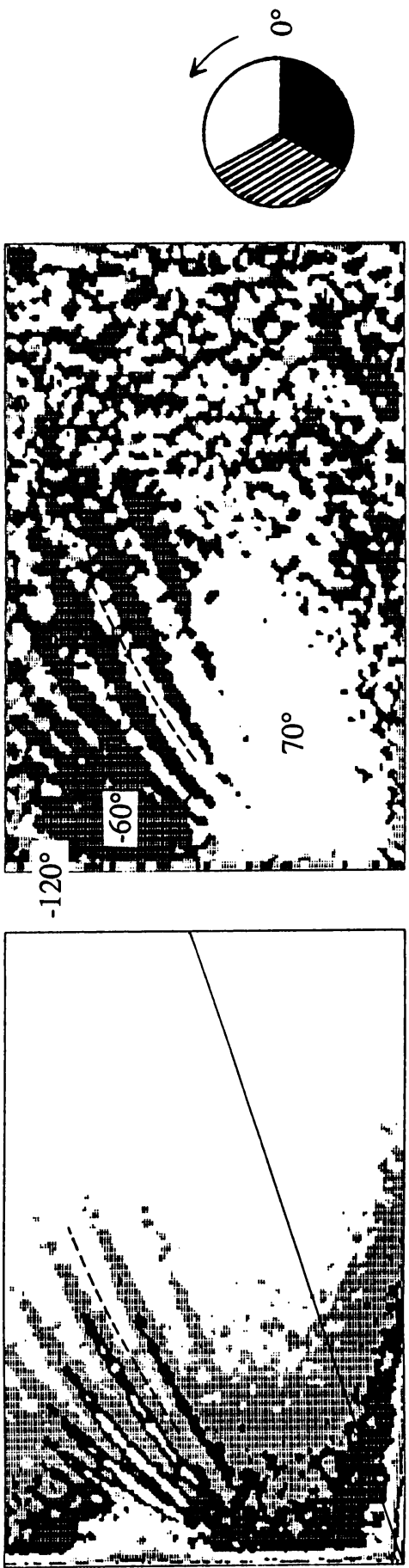

包

ษ 륭혀 붕 훙

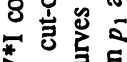
$>$. 它氞寻

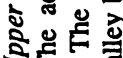
施零

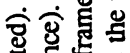

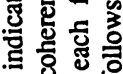
施 을 응 ठ용

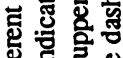
边号

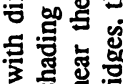

.

解棓

号学文

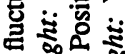
2 을 ญे ฏ. 㑒宫 峞声 药 동응

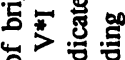

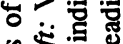
果

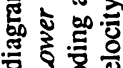
ธิ 苋总总 总这咅

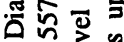

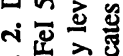
일 


\section{More evanescent waves}

Another reason for continued efforts to better simulate atmospheric waves can be seen next to the region of the phase diagram we have just discussed. As we shall discuss in the next chapter, the low order p-mode ridges exhibit phases which are in satisfactory agreement with theoretical expectation for evanescent waves in an adiabatic gas, namely close to $-90^{\circ}$, in Figure 2. However, side by side with the p-mode ridges, the phase observed in the gaps is drastically different, and probably equal to the value of $+90^{\circ}$ assumed within the trough as discussed in Chapter 3 . This is very clearly visible between the $f-, p_{1^{-}}$and $p_{2}$-modes. With higher order the modes begin to merge, and due to the limited resolving power in our observations the gap between $p_{2}$ and $p_{3}$ is the last one that shows the effect.

We might wonder - since there is much less power in between the ridges - whether the effect is just an illusion due to increased noise in the data. However, incoherent random noise in the phase diagram would have a quite different appearence in our display, with speckles of all three grey scales evenly distributed, whereas the interridge regions as well as the adjacent bottom of the "trough" are fairly uniform in phase.

We have tried to substantiate this statement by computing the coherence of the velocity and brightness fluctuations. The result is displayed in the upper left part of the Figure, decreasing coherence being indicated by darker grey scales. In agreement with our judgement based on the phase displays the coherence is large in the trough and in the adjacent interridge regions, as it is on the ridges themselves. Only at the boundary between the ridge and the gap region the coherence drops sharply for obvious reasons. This can be seen e.g. between the $p_{1}$ and $p_{2}$ ridges.

If the phase anomaly in between the ridges is not an artefact of either the data analysis or seeing (image motion) which we don't believe after several numerical tests we made, we feel encouraged to speculate about a possible solar origin of the weak coherent signal.

It is very unlikely, that the weak continuum under the ridges is caused by short range impulsive events, because in such a case (if the time lag between velocity and brightness is different from zero) one would expect a linear phase dispersion as function of frequency. This is not observed. The only "conservative" assumption left at the moment appears to be the existence of a different type of evanescent waves whose progressive part carries energy downward. A similar type of solution had once been invoked by Hill et al. (1978) to account for the discrepancy between p-mode amplitudes observed as Doppler effect on the disc and the limb oscillations studied with the SCLERA instrument. Here we talk about oscillations being scattered in the inhomogeneous upper atmosphere, with their downward wave fronts directed arbitrarily, which are then no more globally coherent, and therefore uniformly distributed in wavenumber space. In a recent study of the 5-min oscillations Stebbins and Goode (1987) find occasionally a downward phase speed, at certain positions on the sun, especially where the photospheric amplitude is low. It is most interesting to note, that preliminary numerical simulations carried out by Severino and Marmolino (priv. communication) do indeed indicate a V-I phase behaviour that is different from the one observed in the upward solution.

With the present data further hints to the solution of this problem might be expected from studies of the vertical amplitude profile of motions observed between the ridges. A rather more direct approach is obviously to check the cross spectra of velocities measured at different hight levels, and their phases. Unfortunately no such data are available at this moment which have sufficient resolution in both wavenumber and frequency. 


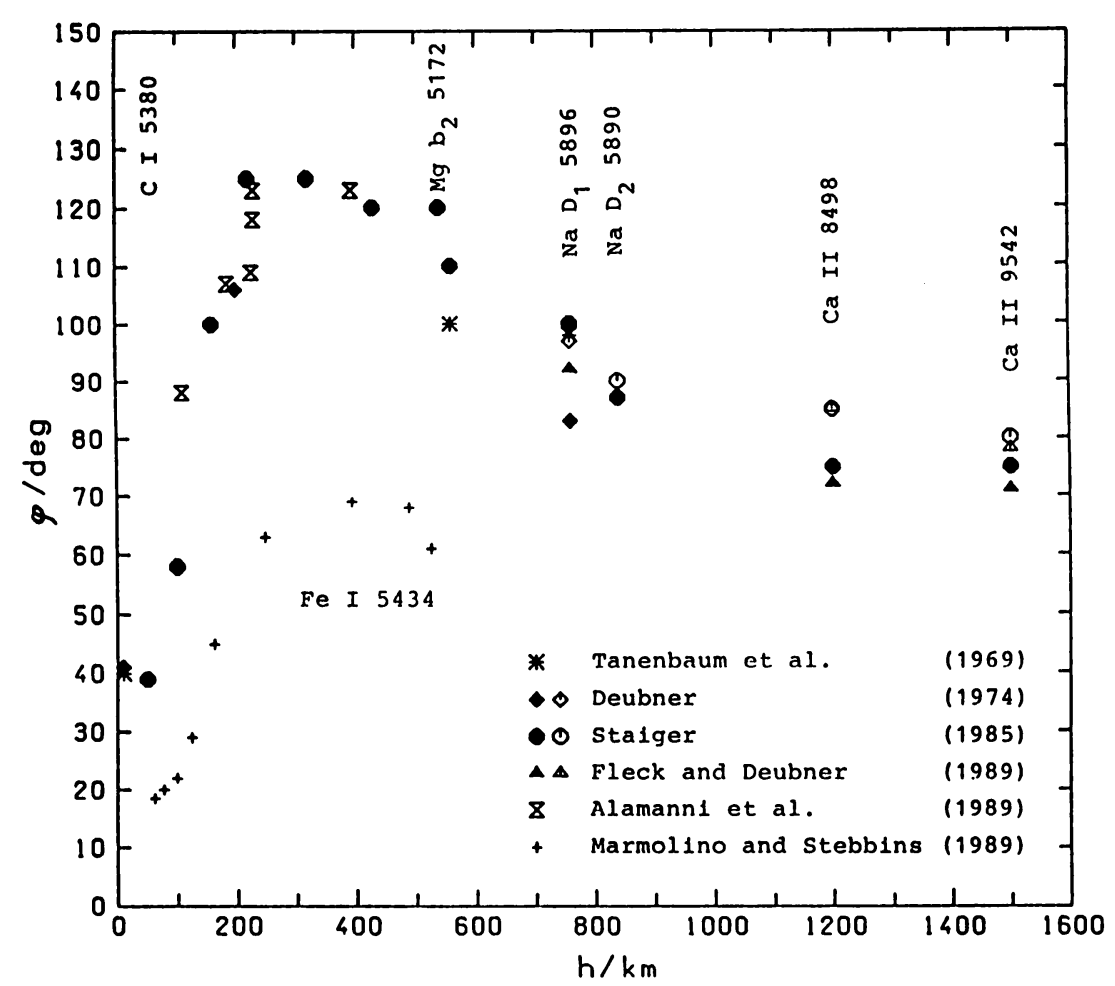

Fig. 3. Phase lag between brightness and velocity in the evanescent frequency range $(5.5 \mathrm{mHz}$ : open diamonds, dots and triangles; $3.3 \mathrm{mHz}$ : all other symbols). Positive phase indicates brightness leading upward velocity. See text for the FeI 5434 results.
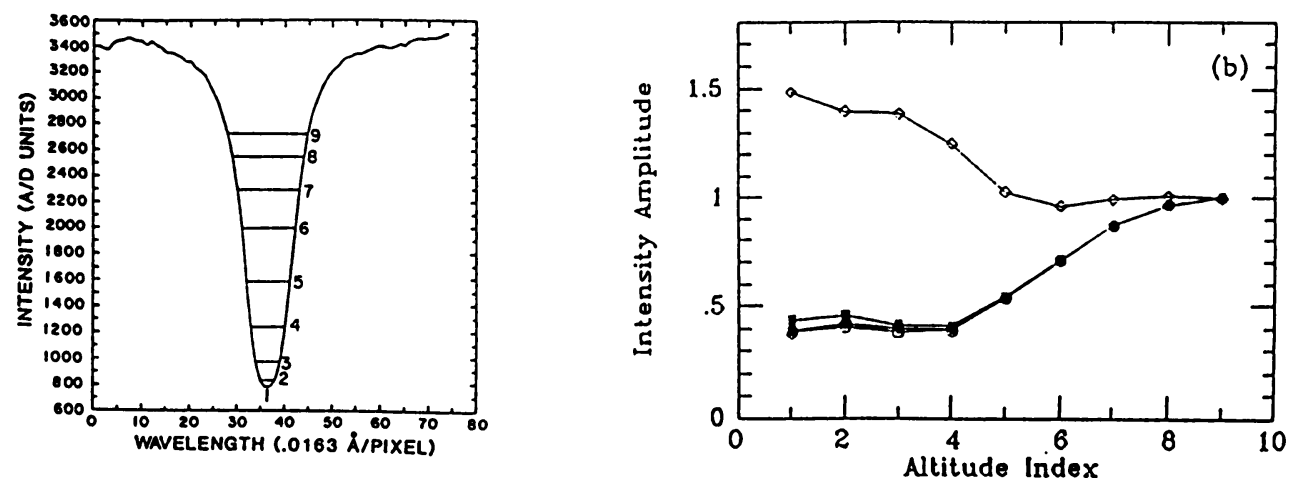

Fig. 4. a: Photometric profile of the FeI 5434 line, with 9 intensity levels at which the V-I phases printed in Fig. 3 with a "+" were determined. Courtesy R.Stebbins and Ph.R.Goode. b: Relative brightness amplitudes of p-mode oscillations in the FeI 5434 line (diamonds: observations; other symbols: various theoretical simulations) as a function of height. The altitude index refers to the levels indicated in Fig. 4a. Courtesy C.Marmolino and R.T.Stcbbins. 


\section{The height dependence of the Velocity-Intensity phases of solar p-modes}

From the theory of evanescent waves we recall that phase values between $90^{\circ}$ and $180^{\circ}$ are predicted in an atmosphere with thermal relaxation. The exact value depends on the relaxation time $\tau$ and on the frequency $\omega$. Above the temperature minimum values close to $90^{\circ}$ (for adiabatic conditions) are expected which should increase continuously to higher values as the photosphere (which is more nearly isothermal) is approached. As we can see in Figure 1, a maximum is reached at a height of about $220 \mathrm{~km}$ above $\tau_{5000}=1$ in the FeI 5930 line; but closer to the photosphere the V-I phase value decreases again. This was already noted long before and has since been studied by several observers whose results are compiled in Figure 3 (with references therein). The Figure evidences a rapid decrease of the phase to values close to $40^{\circ}$ in the lowest $200 \mathrm{~km}$.

It should be kept in mind, that in the evanescent regime phase differences observed between intensity fluctuations at different heights in the atmosphere are almost entirely due to the height dependence of the radiation effects acting on the I signal; since the differential phase lag of the corresponding velocity signals across the full range of heights covered in our diagram is less than $10^{\circ}$, it is also possible to deduce the differential phase lag of the intensity signals from the difference of the corresponding V-I phases.

Recent measurements obtained by Lindsey and Roellig (1987) in the infrared continuum $(\lambda=350 \mu$ and $800 \mu)$ are in excellent agreement with the V-I phases in Figure 3, bearing in mind that the infrared signals are emitted at levels corresponding to the line forming layers of MgI 5172 and NaI 5896 respectively. No special damping mechanism has to be assumed at the higher level to explain the observed vertical phase lag of $25^{\circ}$ to $35^{\circ}$. The value matches closely the corresponding phase lags of the brightness fluctuations in the visual lines at the frequencies between 3 and $6 \mathrm{mHz}$, as may be seen in Figures 1 and 3 . On the contrary, the phase lag observed at the upper level is a consequence of radiative damping at the lower level.

Good agreement exists also with the observations of Jimenez et al. (1989), who report average I-V phases between $-140^{\circ}$ and $-145^{\circ}$ at $3.3 \mathrm{mHz}$. It has to be considered, that their brightness signal is measured in the continuum, i.e. in the lowest photosphere, and is compared with the velocity signal of the potassium resonance line at $770 \mathrm{~nm}$. With the sign convention of Figure 3 (upward velocity positive) the corresponding phase difference is $35^{\circ}$ to $40^{\circ}$, i.e. close to the phase lag measured in the CI 5380 line.

Recent theoretical work on the dynamics of solar p-modes in the atmosphere has been published by Marmolino and Stebbins (1989) and - along the lines of the classical work of Schmieder - by Alamanni et al. (1989) and Cavallini et al. (1987). Neither one of these contributions has been successful in explaining the decrease of V-I phases below $90^{\circ}$ in the photosphere as a property of the p-modes. As in the case of the "missing piece of cake" it was suggested that superposition of the evanescent wave field with convective motions would bias the phase (Schmieder, 1976). Since the phenomenon occurs indeed only close to the photosphere, the argument appears far more convincing in the present context. By the way, the phase values for the FeI 5434 line in Figure 3, taken from the work of Marmolino and Stebbins (1989), have been deduced by averaging the phase signal in the frequency range from 1.3 to $6.3 \mathrm{mHz}$, which includes a large portion of the $2 \mathrm{mHz}$ trough in the phase spectrum, causing a systematic underestimate of these values.

To substantiate or disprove the superposition hypothesis we have constructed $k-\omega$ phase diagrams such as Figure 2 for other spectral lines, CI 5380 and FeI 5576. The search for other sources of a coherent signal with a small V-I phase was unsuccessful in either wavelength; there is just not enough power in the evanescent range at any other spatial scale than that of 
the 5-min oscillations. More importantly, the phases measured in the very ridges follow the same trend with height in the atmosphere, and correspond very closely to those found previously in the frequency spectra, as plotted in Figure 3.

Now, that the observational homework seems to be finished, the solution of the riddle of the p-mode phases can be safely delegated to theory where another problem is already waiting: In their study of the dynamics of p-mode oscillations in the atmosphere Marmolino and Stebbins (1989) have pointed out that the height dependence of the intensity amplitude is seriously at variance with theoretical predictions of all models studied so far (see Figure 4). Rather than the expected decrease of the amplitude of brightness fluctuations, a significant rise of the amplitude with height is observed on the sun; if the simulated signal is calibrated with the observed amplitude at the lowest photospheric level, the difference is as large as a factor of 3 4 at a height of $500 \mathrm{~km}$.

In Figure 5 we have qualitatively depicted the effects on the V-I phase and on the brightness amplitude we have just discussed. $V_{-}$symbolizes upward velocity (blue shift), which lags temperature $\mathrm{T}$ by $90^{\circ}$ in the adiabatic case. If brightness was a good proxi of temperature its phase vector (dashed arrow) would point towards the long dashes with increasing numbers for increasing non-adiabaticity. The limiting case cvidently would be an isothermal medium with a phase angle of $180^{\circ}$ between $V_{-}$and $\mathrm{T}$. In order to obtain a phase angle smaller than $90^{\circ}$, as observed, opacity effects must be important in the photosphere and have to be taken into account in the model calculations.

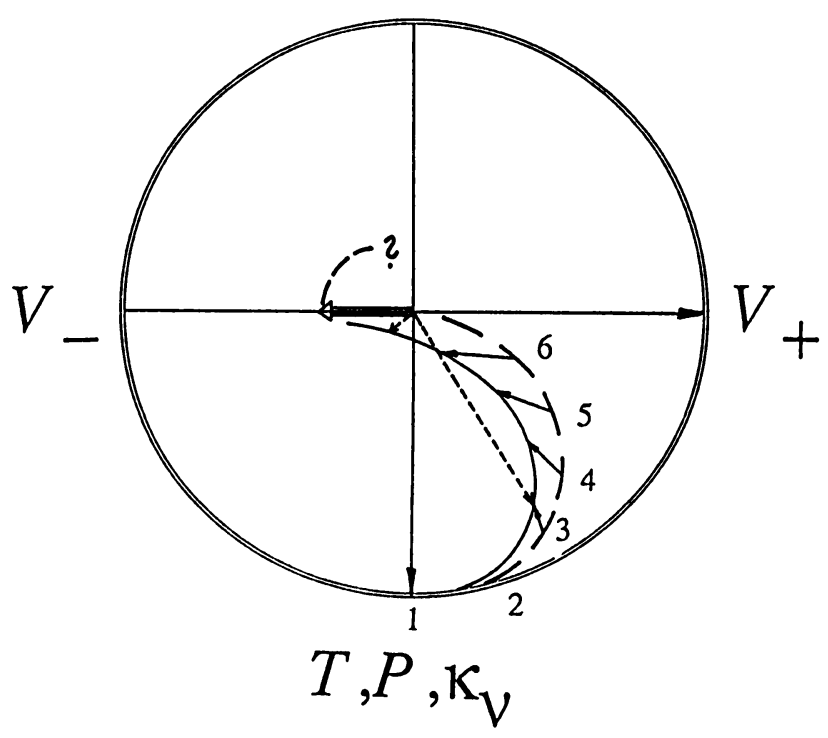

Fig. 5. Schematic phase diagram illustrating the effects of non-adiabaticity and opacity on velocity - brightness phase lags; sec text. 


\section{Conclusion}

Recent theoretical and observational studies have provided an improved understanding of the interaction of convection (in particular granulation) and photospheric oscillations and waves. The hazy picture of the vertical structure of the overshoot region previously derived from coherence studies based on individual high spatial resolution spectra becomes clearer through the analysis of time series, which reveal the existence of coexisting gravity and compression free wave fields, presumably excited by the granules.

On the other hand, the penetration of the global p-mode oscillations into the visible atmosphere, and the whole range of evanescent waves is less well understood than we thought it was. Existing models do not yet reproduce the phase relations between velocity and brightness observed in the low photosphere, nor are the phases in the vicinity of the $f$ - and the low order p-modes in accordance with the customary picture. Apparently we have to regard these regions in the k- $\omega$ diagram as "white spots" which still need a detailed survey by observation, but at the same time we need urgently a more realistic theoretical modelling.

\section{Acknowledgements}

I would like to express my sincere gratitude to the many colleagues who responded to my request of material for this review, and to J. Laufer and B. Fleck who provided valuable practical support at various stages of the research that was incorporated in this report.

\section{References}

Alamanni,N., Bertello,L., Cavallini,F., Ceppatelli,G., Righini,A.: 1989, Astron.Astrophys. (submitted) Brandt,P.N., Scharmer,G.B., Ferguson,S., Shine,R.A., Tarbell,T.D., Title,A.M.: 1988, Nature 335, 238

Brandt,P.N., Scharmer,G.B., Ferguson,S., Shine,R.A., Tarbell,T.D., Title,A.M.: 1989, in High Spatial Resolution Solar Observations (O. von der Lühe, ed.) Sacramento Peak, Sunspot N.M. (in print)

Cavallini,F., Ceppatelli,G., Righini,A., Alamanni,N.: 1987, Astron. Astrophys. 173, 161

Deubner.F.-L.: 1974, Solar Phys. 39, 31

Deubner,F.-L.: 1989, Astron.Astrophys. 216 , 259

Deubner,F.-L., Endler,F., Staiger,J.: 1984, in Oscillations as a Probe of the Sun's Interior (G.Belvedere, L.Paterno, eds.) Mem.Soc.Astron.Ital. 55, 147

Deubner,F.-L., Fleck,B.: 1989, Astron.Astrophys. 213, 423

Dravins,D., Lindegren,L., Nordlund,Å.: 1981, Astron.Astrophys. 96, 345

Evans,J.W., Catalano,C.P.: 1972, Solar Phys. 27, 299

Fleck,B., Deubner,F.-L.: 1989, Astron.Astrophys. (in print)

Frandsen,S.: 1988, in Advances in Helio- and Asteroseismology (J. Christensen-Dalsgaard, S.Frandsen, eds.) D.Reidel, Dordrecht. p. 405

Hill,H.A., Rosenwald,R.D., Caudell,T.P.: 1978, Astrophys.J. 225, 304

Jimenez,A., Pallé,P.L., Roca Cortés,T., Andersen,N.B., Domingo,V., Jones,A., Alvarez,M., Ledezma,E.: 1989, in Seismology of the Sun and Sun-like Stars, Puerto dela Cruz, Tenerife (in print)

Koutchmy,S., Lebecq,D., 1986, Astron.Astrophys. 169, 323

Lindsey,C., Roellig,T.: 1987, Astrophys.J. 313, 877

Lites,B.W., Chipman,E.G.: 1979, Astrophys.J. 231, 570

Marmolino,C., Severino,G.: 1990, in Solar Photosphere: Structure, Convection, and Magnetic Fields (J.O.Stenflo, ed.) 
Marmolino,C., Stebbins,R.T.: 1989, Solar Phys. (submitted)

Mihalas,B.W., Toomre,J.: 1981, Astrophys.J. 249, 349

Nesis,A., Durrant,C.J., Mattig,W.: 1988, Astron.Astrophys. 201, 153

Nordlund,Å.: 1984, in Small Scale Dynamical Processes in Quiet Stellar Atmospheres (S.Keil, ed.) Sunspot N.M. p.181

November,L.J., Toomre,J., Gebbie,K.B., Simon,G.W.: 1981, Astrophys.J. 245, L 123

November,L.J., Simon,G.W., Tarbell,T.D., Title,A.M., Ferguson,S.: 1987, in Theoretical Problems in High Resolution Solar Physics II (G.Athay,D.S. Spicer, eds.) NASA Conf.Publ. 2483R, 121

Noyes,R.W.: 1967, in Aerodynamic Phenomena in Stellar Atmospheres (R.N.Thomas, ed.) New York, Academic Press. p.293

Oda,N.: 1984, Solar Phys. 93, 243

Schmieder,B.: 1976, Solar Phys. 47, 435

Schmieder,B.: 1977, Solar Phys. 54, 269

Schmieder,B.: 1978, Solar Phys. 57, 245

Staiger,J.: 1985, Thesis (Freiburg i.Br.)

Stebbins,R., Goode,Ph.R.: 1987, Solar Phys. 110, 237

Steffen,M.: 1989, in Solar and Stellar Granulation (R.J.Rutten, G.Severino, eds.) Kluwer Acad. Publ., Dordrecht. p.425

Steffen,N., Ludwig,H.G., Krüß,A.: 1989, Astron.Astrophys. 213, 371

Stein,R.F., Nordlund,Å., Kuhn,J.R.: 1989, in Solar and Stellar Granulation (R.J.Rutten, G.Severino, eds.) Kluwer Acad. Publ., Dordrecht. p.381

Strebel,H., Thüring,B.: 1932, Zs.Ap. 5, 348

Tanenbaum,A.S., Wilcox,J.M., Frazier,E.N., Howard,R.: 1969, Solar Phys. 9 , 328

Title,A., Tarbell,T., Simon,G., and the SOUP team: 1986, Adv.Space Res. 6, 253

Wöhl,H., Nordlund,Å.: 1985, Solar Phys. 97, 213 